\title{
Rotating night shift work and adherence to unhealthy lifestyle in predicting risk of type 2 diabetes: results from two large US cohorts of female nurses
}

\author{
Zhilei Shan, ${ }^{1,2}$ Yanping Li, ${ }^{2}$ Geng Zong, ${ }^{2}$ Yanjun Guo, ${ }^{3,4}$ Jun Li, ${ }^{2,3}$ JoAnn E Manson, ${ }^{3,5}$ \\ Frank B Hu, ${ }^{2,3,6}$ Walter C Willett, ${ }^{2,3,6}$ Eva S Schernhammer, ${ }^{3,6,7}$ Shilpa N Bhupathiraju, ${ }^{2,6}$
}

${ }^{1}$ Department of Nutrition and Food Hygiene, Hubei Key Laboratory of Food Nutrition and Safety, Ministry of Education Key Lab of Environment and Health, School of Public Health, Tongji Medical College, Huazhong University of Science and Technology, Wuhan 430030, China

${ }^{2}$ Department of Nutrition, Harvard T.H. Chan School of Public Health, Boston, MA

02115, USA

${ }^{3}$ Department of Epidemiology, Harvard T.H. Chan School of Public Health, Boston, MA, USA

${ }^{4}$ Department of Occupational and Environmental Health,

Ministry of Education Key Lab of Environment and Health, School of Public Health, Tongii Medical College, Huazhong University of Science and Technology,

Wuhan, China

${ }^{5}$ Division of Preventive Medicine, Department of Medicine,

Brigham and Women's Hospital and Harvard Medical School,

Boston, MA, USA

${ }^{6}$ Channing Division of Network

Medicine, Department of

Medicine, Brigham and Women's

Hospital and Harvard Medical

School, Boston, MA, USA

${ }^{7}$ Department of Epidemiology, Center for Public Health,

Medical University of Vienna,

Austria

Correspondence to:

ZShan zshan@hsph.harvard.edu (ORCID 0000-0002-6723-3381)

Additional material is published online only. To view please visit the journal online.

Cite this as: BMJ 2018;363:k4641 http://dx.doi.org/10.1136/bmj.k4641

Accepted: 27 October 2018

\section{ABSTRACT}

OBJECTIVES

To prospectively evaluate the joint association of duration of rotating night shift work and lifestyle factors with risk of type 2 diabetes risk, and to quantitatively decompose this joint association to rotating night shift work only, to lifestyle only, and to their interaction.

DESIGN

Prospective cohort study.

SETTING

Nurses' Health Study (1988-2012) and Nurses' Health Study II (1991-2013).

\section{PARTICIPANTS}

143410 women without type 2 diabetes, cardiovascular disease, or cancer at baseline.

\section{EXPOSURES}

Rotating night shift work was defined as at least three night shifts per month in addition to day and evening shifts in that month. Unhealthy lifestyles included current smoking, physical activity levels below 30 minutes per day at moderate to vigorous intensity, diet in the bottom three fifths of the Alternate Healthy Eating Index score, and body mass index of 25 or above.

\section{MAIN OUTCOME MEASURES}

Incident cases of type 2 diabetes were identified through self report and validated by a supplementary questionnaire.

RESULTS

During 22-24 years of follow-up, 10915 cases of incident type 2 diabetes occurred. The multivariable adjusted hazard ratios for type 2 diabetes were 1.31 (95\% confidence interval 1.19 to 1.44 ) per five year increment of duration of rotating night shift work and 2.30 (1.88 to 2.83 ) per unhealthy lifestyle factor (ever smoking, low diet quality, low physical activity, and overweight or obesity). For the joint association of per five year increment rotating night shift work and per

\section{WHAT IS ALREADY KNOWN ON THIS TOPIC}

Unhealthy lifestyle factors are often reported among rotating night shift workers Both rotating night shift work and unhealthy lifestyle have been associated with a higher risk of type 2 diabetes

\section{WHAT THIS STUDY ADDS}

Duration of rotating night shift work and unhealthy lifestyle were independently and jointly associated with a higher risk of type 2 diabetes

Most cases of type 2 diabetes could be prevented by adherence to a healthy lifestyle, and the benefits could be larger in rotating night shift workers

unhealthy lifestyle factor with type 2 diabetes, the hazard ratio was 2.83 (2.15 to 3.73) with a significant additive interaction ( $P$ for interaction $<0.001$ ). The proportions of the joint association were $17.1 \%$ (14.0\% to $20.8 \%$ ) for rotating night shift work alone, $71.2 \%(66.9 \%$ to $75.8 \%)$ for unhealthy lifestyle alone, and $11.3 \%$ (7.3\% to $17.3 \%)$ for their additive interaction.

\section{CONCLUSIONS}

Among female nurses, both rotating night shift work and unhealthy lifestyle were associated with a higher risk of type 2 diabetes. The excess risk of rotating night shift work combined with unhealthy lifestyle was higher than the addition of risk associated with each individual factor. These findings suggest that most cases of type 2 diabetes could be prevented by adhering to a healthy lifestyle, and the benefits could be greater in rotating night shift workers.

\section{Introduction}

Shift work has progressed in response to changes in economic pressure and greater consumer demand for 24 hour services. ${ }^{1}{ }^{2}$ Increased shift work has many economic advantage, including higher employment, increased services to customers, and improved trade opportunities. ${ }^{3}$ One in five employees in the US works non-standard hours in the evening or night or works rotating shifts. ${ }^{4}$ Health workers make up one third of shift workers, and nurses are the largest group. Nurses have to work shift work schedules to provide services around the clock, which can disrupt social and biological rhythms, increasing health and safety risks. Consequently, the National Institute for Occupational Safety and Health has provided courses since 2015 to train nurses and managers on the risks of shift work as well as strategies to reduce these risks. ${ }^{5}$ In nurses and other shift workers, shift work, especially night shift work, has been associated with a higher risk of chronic diseases, including type 2 diabetes, cardiovascular disease, and several types of cancer. ${ }^{6-8}$

Compelling evidence from well designed observational studies and clinical trials has shown that body weight and lifestyle behaviors, including smoking, diet, and physical activity, can influence risk of type 2 diabetes. ${ }^{9-12}$ Among shift workers, excess adiposity and increased smoking are frequently and consistently reported, ${ }^{13-15}$ whereas the evidence on physical activity is mixed. ${ }^{16}$ Some studies observed alterations in eating habit and specific nutrient intake among shift workers, ${ }^{17-19}$ but the evidence on overall quality of diet across different shift work categories 
is sparse. To our knowledge, no previous study has examined the joint associations of duration of rotating night shift work and unhealthy lifestyle factors with risk of type 2 diabetes or evaluated their potential interactions. ${ }^{316}$

Therefore, we analyzed data for participants in two large prospective cohorts to test the hypothesis that rotating night shift work and unhealthy lifestyle factors were jointly associated with risk of type 2 diabetes. We then quantitatively calculated the proportions of this joint association for rotating night shift work, unhealthy lifestyle factors, and their interaction.

\section{Methods}

\section{Study population}

We used data from the Nurses' Health Study (NHS) and NHS II. The NHS began in 1976 when 121701 female registered US nurses, aged 30 to 55 years, responded to a baseline questionnaire. The NHS II started in 1989 and included 116677 female registered US nurses, aged 25 to 42 years. In the NHS and NHS II, baseline and follow-up questionnaires were sent to participants every two years to update medical and lifestyle information over the follow-up period. Participants completed an initial food frequency questionnaire in 1980 for NHS and 1991 for NHS II. Food frequency questionnaires were updated approximately every four years thereafter. The follow-up rates exceeded $90 \%$ in both cohorts.

We used 1988 as baseline for the NHS and 1991 for NHS II, when information on both rotating night shift work and all lifestyle factors was first ascertained. At baseline, we excluded participants with a self report of diabetes, coronary heart disease, stroke, or cancer, as the diagnosis of these diseases could potentially lead to changes in their lifestyle or the shift work routine. We further excluded participants with missing data on shift work and important covariates including age, diet, physical activity, smoking status, or body weight. The final analysis included 55324 participants from the NHS and 88086 participants from the NHS II. The details of the exclusions are shown in supplementary figure A.

\section{Ascertainment of rotating night shift work}

In 1988 the NHS participants were asked the following question: "What is the total number of years during which you worked rotating night shifts (at least three nights per month in addition to days/evenings in that month)?", with eight pre-specified response categories (never, 1-2, 3-5, 6-9, 10-14, 15-19, 20-29, and $\geq 30$ years). In 1989 the NHS II participants were asked the same question with seven pre-specified response categories (never, 1-2, 3-5, 6-9, 10-14, 15-19, and $\geq 20$ years). We updated this information in 1991, 1993, 1997, 2001, 2005, 2007, and 2009 in NHS II. In 1991, 1993, 1997, 2007, and 2009 we collected information on the total number of months during which the nurse had worked rotating night shifts in the previous two years, with pre-specified response categories (none,
1-4, 5-9, 10-14, 15-19, and $\geq 20$ months). Additionally, in 2001 we asked about duration of rotating night shifts in 1993-95, 1995-97, 1997-99, and 1999-2001. In 2005 we collected data on the duration of rotating night shifts in 2001-03 and 2003-05. We assigned and added together midpoint values in years and calculated the cumulative years of rotating night shifts for the NHS II women. The analyses reported here used baseline assessments of lifetime shift work history in the NHS and cumulative shift work exposure through 2009 in the NHS II.

\section{Ascertainment of lifestyle factors}

We included four lifestyle factors: diet, physical activity, smoking, and body mass index. Diet was assessed every four years with a validated food frequency questionnaire that asked how often, on average, a participant had consumed a particular amount of a specific type of food during the previous year. $^{20}$ Intakes of nutrients were calculated by multiplying the frequency of consumption of each unit of food by its nutrient content. Quality of diet was assessed using the Alternate Healthy Eating Index (AHEI) score. Briefly, points were assigned for intake of each component on a scale from 0 to 10 , with 10 indicating adherence to the recommended levels of servings per day. We included 10 components of the index in our diet score: high intakes of vegetables, fruit, nuts, whole grains, polyunsaturated fatty acids, and long chain omega-3 fatty acids; and low intakes of red and processed meats, sugar sweetened drinks, trans fat, and sodium. In our previous analyses, the AHEI score was strongly associated with the risk of type 2 diabetes. $^{21}$

Levels of physical activity were investigated using a validated questionnaire, which was updated every two years. ${ }^{22}$ We estimated the number of hours that participants spent each week doing moderate to vigorous activities (including brisk walking) requiring at least the expenditure of at least 3 metabolic equivalent units (MET) per hour. The validity and reproducibility of the questionnaire has been evaluated in a representative sample of participants $(n=147)$ in the NHS II and reported previously. ${ }^{23}$ The overall correlation between physical activity reported on recalls and on questionnaire was 0.79 , and the correlation between moderate to vigorous physical activity reported in diaries and on questionnaires was 0.62 . $^{23}$

Smoking habits were categorized as never smoker, former smoker, or current smoker (including the number of cigarettes smoked per day) and updated every two years. Body mass index was calculated as self reported weight $(\mathrm{kg})$ divided by height in meters squared. In our validation study, the correlation between self reported and technician measured weight was $0.97 .^{24}$

\section{Definition of unhealthy and healthy lifestyle}

We defined low risk lifestyle factors as normal weight (body mass index $\geq 18.5$ and $<25$ ), not smoking, 
physical activity levels of at least 30 minutes a day at moderate to vigorous intensity, and AHEI score in the upper two fifths. ${ }^{25}$ For each unhealthy lifestyle factor, participants received a score of 0 if they met the criterion for low risk or 1 otherwise.

\section{Assessment of other confounders}

Information on potential confounders was assessed and updated every other year via the questionnaires throughout follow-up. This information included age, marital status, living status, use of aspirin, use of multivitamins, menopausal status and postmenopausal hormone use, oral contraceptive use, prevalent hypertension and hyperlipidemia, and drug treatment for high blood pressure and high lipid concentrations. Alcohol use was assessed and updated every four years from the food frequency questionnaires.

\section{Ascertainment of incident type 2 diabetes}

In both cohorts, cases of type 2 diabetes were identified by self report and confirmed by a validated supplementary questionnaire. We considered a case of type 2 diabetes to be confirmed if at least one of the following was reported on the supplementary questionnaire according to the National Diabetes Data Group criteria: one or more classic symptoms (excessive thirst, polyuria or frequent urination, weight loss, hunger) plus fasting plasma glucose concentrations of at least $7.8 \mathrm{mmol} / \mathrm{L}$ or random plasma glucose concentrations of at least $11.1 \mathrm{mmol} / \mathrm{L}$; at least two elevated plasma glucose concentrations on different occasions (fasting plasma glucose concentrations of at least $7.8 \mathrm{mmol} / \mathrm{L}$, random concentrations of at least $11.1 \mathrm{mmol} / \mathrm{L}$, and/or two hour blood glucose concentrations of at least $11.1 \mathrm{mmol} / \mathrm{L}$ during oral glucose tolerance testing) in the absence of symptoms; or treatment with hypoglycemic drugs (insulin or oral hypoglycemic agent). ${ }^{26}$ For cases identified after 1998, we applied the American Diabetes Association's criteria, in which the threshold for fasting plasma glucose changed from $7.8 \mathrm{mmol} / \mathrm{L}$ to $7.0 \mathrm{mmol} / \mathrm{L} .{ }^{27}$ The validity of the supplemental questionnaire was established by a review of medical reports. ${ }^{28}$ In a random sample of 62 cases in the NHS that were confirmed by the supplementary questionnaire, 61 (98\%) cases were reconfirmed after medical records were reviewed by an endocrinologist blinded to the supplementary questionnaire. ${ }^{28}$

\section{Statistical analysis}

We age standardized the participants' baseline characteristics except age, according to duration of rotating night shift work, to allow populations to be compared. Participants contributed person time from the return of the baseline questionnaire (NHS, 1988; NHS II, 1991) until the date of diagnosis of type 2 diabetes, death, loss to follow-up, or the end of the follow-up period (30 June 2012 for the NHS and 30 June 2013 for NHS II), whichever came first. We used multivariable time dependent Cox proportional hazards models to estimate hazard ratios and 95\% confidence intervals for the associations between shift work alone and in combination with unhealthy lifestyle factors and incident type 2 diabetes. We used the two year follow-up intervals to construct the time intervals. If a participant quit rotating night shift work, we used the cumulative shift work duration in the last interval.

For the association between duration of rotating night shift work and risk of type 2 diabetes, we selected participants without a history of rotating night shift work as the reference group. A linear trend was quantified across rotating night shift work duration categories by assigning the median value to each category and modeling this variable as a continuous variable. In multivariable analysis, we adjusted for several confounding factors including ethnicity, family history of diabetes, living alone or with others, marital status, menopausal status and postmenopausal hormone therapy use, oral contraceptive use (NHS II only), smoking status, alcohol consumption, physical activity, and the AHEI. In a subsequent model, we additionally adjusted for body mass index.

We examined the associations of the overall unhealthy lifestyle score and the individual lifestyle factors with risk of type 2 diabetes by adjusting for the covariates listed above. Each individual lifestyle factor was mutually adjusted for each other. We then classified participants according to the joint categories of rotating night shift work duration and the number of unhealthy lifestyle factors. We used updated levels of lifestyle factors to calculate the unhealthy lifestyle score from the most recent questionnaire. Using multiplicative and additive interaction analyses, we evaluated whether the associations between rotating night shift work duration and type 2 diabetes differed by number of unhealthy lifestyle factors. ${ }^{29}{ }^{30} \mathrm{We}$ tested for the presence of multiplicative interaction by including a cross-product term between rotating night shift work duration and unhealthy lifestyle score in our fully adjusted multivariable model. We then compared the -2 log likelihood values in models with and without the cross-product interaction term. To assess additive interaction between rotating night shift work duration and unhealthy lifestyle on risk of type 2 diabetes, we considered duration of rotating night shift work and the number of unhealthy lifestyle factors as two continuous variables. The relative excess risk due to interaction was assessed as an index of additive interaction. ${ }^{31}$ We also examined the decomposition of the joint effect: the proportion attributable to rotating night shift work alone, to unhealthy lifestyle alone, and to the interaction. ${ }^{31}$ Briefly, on the hazard ratio scale, we decomposed the joint excess relative risk for both exposures $\left(\mathrm{HR}_{11}-1\right)$ into the excess relative risk for shift work alone $\left(\mathrm{HR}_{01}-1\right)$, unhealthy lifestyle alone $\left(\mathrm{HR}_{10}-1\right)$, and relative excess risk due to interaction (RERI). Specifically, we have $\mathrm{HR}_{11}-1=\left(\mathrm{HR}_{01}-1\right)+\left(\mathrm{HR}_{10}-1\right)+\mathrm{RERI}$. We then likewise calculated the proportion of the joint effect due to shift work alone $\left(\mathrm{HR}_{01}-1\right) /\left(\mathrm{HR}_{11}-1\right)$; due to unhealthy 
lifestyle alone $\left(\mathrm{HR}_{10}-1\right) /\left(\mathrm{HR}_{11}-1\right)$; and due to their additive interaction, $\mathrm{RERI} /\left(\mathrm{HR}_{11}-1\right)$.

Owing to differences in age, follow-up time, and the questionnaires in the two cohorts, we did all analyses separately in each cohort to achieve better control of confounding. We pooled the hazard ratios from the multivariable adjusted models in each cohort to obtain a summarized risk estimate by using an inverse variance weighted, random effect meta-analysis. We used the Cochrane $\mathrm{Q}$ statistic and the $\mathrm{I}^{2}$ statistic to examine the heterogeneity of associations between the cohorts.

We did several sensitivity analyses to test the robustness of our results. Firstly, we additionally adjusted for prevalent hypertension and hyperlipidemia and for drug treatment for high blood pressure and high lipid concentrations. Secondly, we did analyses to assess the joint association of duration of rotating night shift work and unhealthy lifestyle with risk of symptomatic type 2 diabetes, defined as participants who reported at least one symptom of diabetes. Thirdly, to test whether our results were biased by stopping updating selectively, we used baseline levels of lifestyle factors to calculate the unhealthy lifestyle score. Fourthly, because body mass index had a strong and positive association with risk of type 2 diabetes, we removed it from our lifestyle factors score. Fifthly, we created a weighted unhealthy lifestyle score in which each unhealthy lifestyle factor was weighted on the basis of the multivariable regression coefficient of its association with type 2 diabetes. Finally, we used fixed effects, rather than random effects, models. We used SAS 9.3 for UNIX for all analyses and set statistical significance at a two tailed $\mathrm{P}$ value below 0.05 .

\section{Patient involvement}

No patients were involved in setting the research question or the outcome measures, nor were they involved in the design and implementation of the study. There are no plans to involve patients in dissemination.

\section{Results}

Table 1 shows age and the age adjusted characteristics of study participants at baseline according to categories of duration of rotating night shift work. In both cohorts, compared with women with no history of rotating night shift work, those with more years spent in rotating night shift work were more likely to be current smokers and to have higher body mass index. With increasing duration of rotating night shift work, the participants were older in the NHS; a greater proportion of women were unmarried and lived alone in the NHS II. No appreciable differences in quality of diet and amount of physical activity were apparent across durations of rotating night shift work.

We documented 5474 incident cases of type 2 diabetes during 1139597 person years of follow-up in the NHS and 5441 cases during 1778721 person years of follow-up in NHS II. We observed a positive

\begin{tabular}{|c|c|c|c|c|}
\hline \multirow[b]{2}{*}{ Characteristics } & \multicolumn{4}{|c|}{ Duration of rotating night shift work (years) } \\
\hline & Never & $1-5$ & $5-9$ & $\geq 10$ \\
\hline \multicolumn{5}{|l|}{ Nurses' Health Study $1988(n=55324)$} \\
\hline Total & $22603(41)$ & $23008(42)$ & $3677(7)$ & $6036(11)$ \\
\hline Mean (SD) age, years & $53.4(7.1)$ & $53.7(7.1)$ & $54.5(7.0)$ & $55.5(6.9)$ \\
\hline Mean (SD) body mass index & $25.1(4.6)$ & $25.2(4.6)$ & $25.8(5.0)$ & $26.4(5.1)$ \\
\hline Mean (SD) total energy intake, kcal/day & $1751(520)$ & $1788(523)$ & $1777(540)$ & $1787(546)$ \\
\hline Mean (SD) Alternate Healthy Eating Index & $45.9(10.5)$ & $46.3(10.5)$ & $46.3(10.5)$ & $45.5(10.1)$ \\
\hline Mean (SD) alcohol intake, g/day & $6.2(10.7)$ & $6.4(10.6)$ & $6.0(10.5)$ & $5.4(10.2)$ \\
\hline Mean (SD) moderate to vigorous intensity exercise, $\mathrm{h} /$ week & $1.9(3.3)$ & $2.2(3.5)$ & $2.0(3.3)$ & $2.1(3.6)$ \\
\hline White ethnicity & $22151(98)$ & $22548(98)$ & $3603(98)$ & $5855(97)$ \\
\hline Current smoker & $3843(17)$ & $3911(17)$ & $772(21)$ & $1388(23)$ \\
\hline Married & $16726(74)$ & $17026(74)$ & $2611(71)$ & $4165(69)$ \\
\hline Living alone & $2260(10)$ & $2301(10)$ & $404(11)$ & $724(12)$ \\
\hline Postmenopausal & $15822(70)$ & $16106(70)$ & $2611(71)$ & $4467(74)$ \\
\hline Family history of diabetes & $6329(28)$ & $6672(29)$ & $1140(31)$ & $1871(31)$ \\
\hline \multicolumn{5}{|l|}{ Nurses' Health Study II $1991(n=88086)$} \\
\hline Total & $30100(34)$ & $44720(51)$ & $8242(9)$ & $5024(6)$ \\
\hline Mean (SD) age, years & $36.3(4.7)$ & $35.9(4.7)$ & $35.9(4.5)$ & $37.7(3.9)$ \\
\hline Mean (SD) body mass index & $24.2(5.0)$ & $24.4(5.2)$ & $25.2(5.7)$ & $25.9(6.0)$ \\
\hline Mean (SD) total energy intake, kcal/day & $1765(536)$ & $1794(548)$ & $1803(560)$ & $1828(576)$ \\
\hline Mean (SD) Alternate Healthy Eating Index & $43.6(10.5)$ & $44.3(10.5)$ & $44.1(10.4)$ & $43.3(10.5)$ \\
\hline Mean (SD) alcohol intake, g/day & $3.0(6.0)$ & $3.3(6.2)$ & $3.2(6.0)$ & $2.9(5.8)$ \\
\hline Mean (SD) moderate to vigorous intensity exercise, $\mathrm{h} /$ week & $2.2(3.5)$ & $2.4(3.8)$ & $2.6(3.9)$ & $2.6(4.1)$ \\
\hline White ethnicity & $28896(96)$ & $42931(96)$ & $7830(95)$ & $4773(95)$ \\
\hline Current smoker & $3311(11)$ & $5366(12)$ & $1154(14)$ & $854(17)$ \\
\hline Married & $24381(81)$ & $34882(78)$ & $5934(72)$ & $3567(71)$ \\
\hline Living alone & $2107(7)$ & $3578(8)$ & $824(10)$ & $603(12)$ \\
\hline Postmenopausal & $903(3)$ & $1342(3)$ & $247(3)$ & $201(4)$ \\
\hline Family history of diabetes & $4515(15)$ & $7155(16)$ & $1401(17)$ & $955(19)$ \\
\hline
\end{tabular}




\begin{tabular}{|c|c|c|c|c|c|}
\hline \multirow[b]{2}{*}{ Cohorts } & \multicolumn{4}{|c|}{ Duration of rotating night shift work (years) } & \multirow[b]{2}{*}{$P$ value for trend } \\
\hline & Never & $1-5$ & $5-9$ & $\geq 10$ & \\
\hline \multicolumn{6}{|l|}{ Nurses' Health Study (1988-2012) } \\
\hline Cases/person years & $2068 / 470118$ & $2200 / 477555$ & $405 / 73963$ & $801 / 117961$ & - \\
\hline Incidence rate (per $10^{5}$ person years) & 440 & 461 & 548 & 679 & - \\
\hline Age adjusted & 1.00 (reference) & $1.04(0.98$ to 1.11$)$ & $1.24(1.12$ to 1.38$)$ & $1.53(1.41$ to 1.66$)$ & $<0.001$ \\
\hline Multivariable adjusted* & 1.00 (reference) & 1.04 (0.98 to 1.10$)$ & 1.18 (1.06 to 1.31$)$ & 1.39 (1.28 to 1.51$)$ & $<0.001$ \\
\hline Further adjusted for lifestylet & 1.00 (reference) & $1.05(0.99$ to 1.12$)$ & $1.18(1.06$ to 1.31$)$ & $1.39(1.28$ to 1.51$)$ & $<0.001$ \\
\hline Further adjusted for body mass index $\ddagger$ & 1.00 (reference) & $1.02(0.96$ to 1.09$)$ & 1.07 (0.96 to 1.19$)$ & $1.16(1.06$ to 1.26$)$ & $<0.001$ \\
\hline \multicolumn{6}{|l|}{ Nurses’ Health Study II (1991-2013) } \\
\hline Cases/person years & $1373 / 546331$ & $2583 / 886635$ & $733 / 198018$ & $752 / 147737$ & - \\
\hline Incidence rate (per $10^{5}$ person years) & 251 & 291 & 370 & 509 & - \\
\hline Age adjusted & 1.00 (reference) & $1.18(1.11$ to 1.26$)$ & $1.50(1.37$ to 1.64$)$ & 1.81 (1.65 to 1.98$)$ & $<0.001$ \\
\hline Multivariable adjusted* & 1.00 (reference) & 1.15 (1.08 to 1.23$)$ & $1.39(1.27$ to 1.52$)$ & $1.56(1.43$ to 1.71$)$ & $<0.001$ \\
\hline Further adjusted for lifestylet & 1.00 (reference) & $1.16(1.09$ to 1.24$)$ & $1.38(1.26$ to 1.51$)$ & $1.54(1.41$ to 1.69$)$ & $<0.001$ \\
\hline Further adjusted for body mass index $\ddagger$ & 1.00 (reference) & $1.07(1.00$ to 1.14$)$ & $1.11(1.02$ to 1.22$)$ & 1.17 (1.07 to 1.28$)$ & $<0.001$ \\
\hline \multicolumn{6}{|l|}{ Pooled results } \\
\hline Age adjusted & 1.00 (reference) & $1.11(0.98$ to 1.25$)$ & $1.37(1.14$ to 1.64$)$ & $1.66(1.41$ to 1.96$)$ & $<0.001$ \\
\hline Multivariable adjusted* & 1.00 (reference) & $1.09(0.99$ to 1.21$)$ & $1.28(1.09$ to 1.51$)$ & $1.47(1.32$ to 1.65$)$ & $<0.001$ \\
\hline Further adjusted for lifestyle† & 1.00 (reference) & 1.11 (1.00 to 1.22$)$ & $1.28(1.10$ to 1.49$)$ & $1.46(1.33$ to 1.62$)$ & $<0.001$ \\
\hline Further adjusted for body mass index $\neq$ & 1.00 (reference) & 1.04 (1.00 to 1.09$)$ & 1.09 (1.02 to 1.17$)$ & $1.16(1.09$ to 1.24$)$ & $<0.001$ \\
\hline \multicolumn{6}{|c|}{$\begin{array}{l}\text { Multivariable adjusted hazard ratios were estimated from Cox proportional hazards models. All covariates, except ethnicity and family history of diabetes, were time varying. } \\
\text { *Adjusted for age, calendar year, ethnicity (white, African-American, Hispanic, or Asian), marital status (married, divorced/separate/single, or widowed); living status (alone or not), family history } \\
\text { of diabetes (yes/no), menopausal status (premenopausal or postmenopausal; never, past, or current menopausal hormone use), oral contraceptive use (never, past, or current use (NHS II only)), } \\
\text { alcohol drinking (0, 0.1-4.9, 5.0-14.9, 15.0-19.9, 20.0-29.9, or } \geq 30 \mathrm{~g} / \text { day), and total energy intake (fifths). } \\
\text { tFurther adjusted for smoking status (never smoker, former smoker, current smoker: } 1-14,15-24 \text {, or } \geq 25 \text { cigarettes/day), physical activity (0, } 0.1-1.0,1.0-3.5,3.5-6.0, \text { or } \geq 6 \text { hours/week), and } \\
\text { Alternate Healthy Eating Index score (fifths). } \\
\text { fFurther adjusted for body mass index (<21, } 21-24.9,25-29.9,30-35 \text {, or } \geq 35 \text { ). }\end{array}$} \\
\hline
\end{tabular}

association between duration of rotating night shift work and risk of type 2 diabetes in both cohorts. Compared with women without rotating night shift work, the pooled multivariable adjusted hazard ratios for women with $1-5,5-9$, and 10 or more years of rotating night shift work were 1.11 (95\% confidence

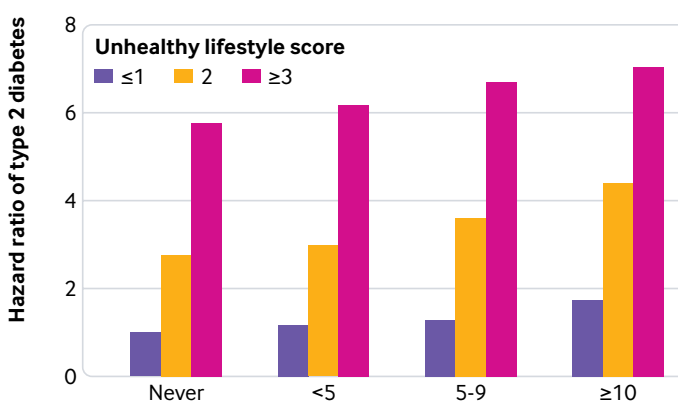

Duration of rotating night shift work (year)

Fig 1 | Pooled multivariable adjusted hazard ratios of type 2 diabetes according to joint categories of rotating night shift work duration and number of unhealthy lifestyle factors. Multivariable adjusted for age, calendar year, ethnicity (white, African-American, Hispanic, or Asian), marital status (married, divorced/separate/single, or widowed), living status (alone or not), family history of diabetes (yes/no), menopausal status (premenopausal or postmenopausal; never, past, or current menopausal hormone use), oral contraceptive use (never, past, or current use (NHS II only)), alcohol drinking (0, 0.1-4.9, 5.0-14.9, 15.0-19.9, 20.0-29.9, or $\geq 30 \mathrm{~g} /$ day), and total energy intake (fifths). All covariates, except ethnicity and family history of diabetes, were time varying. Unhealthy lifestyles include current smoking, physical activity levels $<30 \mathrm{~min} /$ day at moderate to vigorous intensity, diet in bottom three fifths of Alternate Healthy Eating Index score, and body mass index $\geq 25$ interval 1.00 to 1.22 ), 1.28 (1.10 to 1.49 ), and 1.46 (1.33 to 1.62 ) ( $P$ for trend <0.001) (table 2). When we further adjusted for updated body mass index, the association was attenuated but remained significant for rotating night shift work duration of at least five years (hazard ratio 1.04 (1.00 to 1.09) for 1-5 years; 1.09 (1.02 to 1.17) for 5-9 years; 1.16 (1.09 to 1.24) for $\geq 10$ years; $P$ for trend $<0.001$ ).

When we examined the association of individual lifestyle factors with risk of type 2 diabetes, current smoking and a higher body mass index were positively associated with risk of type 2 diabetes (supplementary table A). However, a higher AHEI score and a higher level of moderate to vigorous physical activity were both associated with a lower risk of type 2 diabetes (supplementary table A). When considered together, compared with participants with one or fewer unhealthy lifestyle factors, a combination of at least three unhealthy lifestyle factors was associated with more than a fivefold risk (hazard ratio 5.39, 3.65 to 7.95) for type 2 diabetes (supplementary table A).

When we examined the association of joint categories of duration of rotating night shift work and the number of unhealthy lifestyle factors, within each category of rotating night shift work duration, each additional unhealthy lifestyle factor was consistently associated with a higher risk of type 2 diabetes. Across all levels of unhealthy lifestyle factors, we documented a consistent and graded increasing risk of type 2 diabetes with increasing duration of rotating night shift work. Compared with the reference group (those having no exposure to rotating night shift work and one or fewer unhealthy lifestyle factors), the multivariable adjusted hazard ratio for type 2 diabetes among women with at least 10 years of rotating night 
shift work duration and with at least three unhealthy lifestyle factors was 7.04 (5.29 to 9.37) (fig 1). Tests for multiplicative interactions were not significant ( $\mathrm{P}>0.67)$.

We documented a significant additive interaction between duration of rotating night shift work and number of unhealthy lifestyle factors $(\mathrm{P}<0.001)$. The multivariable adjusted hazard ratios for type 2 diabetes were 1.31 (1.19 to 1.44 ) per five year increment of rotating night shift work duration, 2.30 (1.88 to 2.83) per unhealthy lifestyle factor, and 2.83 (2.15 to 3.73) for their joint effect, with a relative excess risk due to interaction of 0.20 ( 0.09 to 0.48 ) (table 3$)$. The attributable proportions of the joint effect were $17.1 \%$ (95\% confidence interval $14.0 \%$ to $20.8 \%$ ) for rotating night shift work alone, $71.2 \%$ (66.9\% to $75.8 \%)$ for unhealthy lifestyle alone, and $11.3 \%$ (7.3\% to $17.3 \%)$ for their interaction. The relative excess risk due to the interaction was relatively higher in the NHS II $(0.32$, 0.21 to 0.42$)$ than in the NHS $(0.13,0.09$ to 0.17$)$.

In sensitivity analyses, the joint associations of rotating night shift work and lifestyle risk factors with type 2 diabetes were slightly attenuated, but they remained significant after additional adjustment for prevalent hypertension, hyperlipidemia, and drug treatment for high blood pressure and high lipid concentrations (supplementary table B). When we restricted cases of type 2 diabetes to those with symptoms, results were similar to those from the main joint analysis (supplementary figure B). When we used the baseline lifestyle score in place of the updated lifestyle score, the joint association was attenuated but remained consistent with the main joint analysis (supplementary figure C). Results were similar when we use the weighted lifestyle score by assigning weights to each lifestyle factor (supplementary table $\mathrm{C}$ ). When we removed body mass index from the unhealthy lifestyle score, the joint associations of rotating night shift work and lifestyle risk factors with type 2 diabetes were observed among the overall, body mass index below 25 , and body mass index 25 or higher populations, without evidence of an additive or multiplicative interaction (supplementary figure D). When we used fixed effects instead of random effects models, results were not materially altered.

\section{Discussion}

In two large cohorts of US nurses, duration of rotating night shift work and unhealthy lifestyle (current smoking, a low quality of diet, low physical activity levels, and overweight or obesity) were independently and jointly associated with a higher risk of type 2 diabetes. The proportions of the joint association were $17 \%$ for duration of rotating night shift work alone, $71 \%$ for unhealthy lifestyle alone, and $11 \%$ for an additive interaction between rotating night shift work duration and unhealthy lifestyle.

\section{Comparison with other studies}

Our findings highlight the additive interaction between rotating night shift work and unhealthy lifestyle on risk of type 2 diabetes. In particular, if both rotating night shift work and unhealthy lifestyle were present, this would result in an additional 11\% of type 2 diabetes cases. From a public health standpoint, because 71\% of the joint effect could be attributed to an unhealthy lifestyle, our findings underscore the importance of maintaining a healthy lifestyle. Our results are consistent with previous findings from our group,,$^{9} 25$ as well as with public health recommendations. ${ }^{32}$ In this analysis, the main effects of rotating night shift work, unhealthy lifestyles, and their joint interaction were relatively stronger in the NHS II than in the NHS, which may partly be because participants in the NHS were older than those in the NHS II. Consistent with our findings, a previous study also found that the association between years of shift work and type 2 diabetes was stronger in younger than older participants. ${ }^{33}$ Similarly, we also observed that the effects of modifiable lifestyle factors on mitigating type 2 diabetes and hypertension tended to decrease

\begin{tabular}{|c|c|c|c|c|}
\hline & Nurses' Health Study & Nurses' Health Study II & Pooled results* & P for heterogeneity $\dagger$ \\
\hline \multicolumn{5}{|l|}{ Main effects, hazard ratio $(95 \% \mathrm{Cl})$} \\
\hline Shift work duration (per 5 years) & $1.25(1.15$ to 1.37$)$ & $1.38(1.24$ to 1.54$)$ & $1.31(1.19$ to 1.44$)$ & 0.17 \\
\hline Unhealthy lifestyleł (per unit increase) & 2.08 (1.98 to 2.18$)$ & $2.56(2.43$ to 2.70$)$ & $2.30(1.88$ to 2.83$)$ & $<0.001$ \\
\hline Joint effect & $2.46(2.37$ to 2.55$)$ & $3.26(3.15$ to 3.37$)$ & $2.83(2.15$ to 3.73$)$ & $<0.001$ \\
\hline \multicolumn{5}{|c|}{ Relative excess risk $(95 \% \mathrm{Cl})$ due to interaction } \\
\hline Relative excess risk due to interaction & $0.13(0.09$ to 0.17$)$ & $0.32(0.21$ to 0.42$)$ & $0.20(0.09$ to 0.48$)$ & $<0.001$ \\
\hline P value & $<0.001$ & $<0.001$ & $<0.001$ & - \\
\hline \multicolumn{5}{|l|}{ Attributable proportion, $\%(95 \% \mathrm{Cl})$} \\
\hline Shift work & $17.3(11.9$ to 22.7$)$ & $16.9(12.7$ to 21.1$)$ & 17.1 (14.0 to 20.8$)$ & 0.91 \\
\hline Unhealthy lifestyleł & 73.7 (68.1 to 79.2$)$ & $69.1(64.5$ to 73.7$)$ & $71.2(66.9$ to 75.8$)$ & 0.22 \\
\hline Additive interaction & $9.0(7.2$ to 10.9$)$ & $14.0(11.4$ to 16.5$)$ & $11.3(7.3$ to 17.3$)$ & 0.002 \\
\hline \multicolumn{5}{|c|}{$\begin{array}{l}\text { Multivariable adjusted for age, calendar year, ethnicity (white, African-American, Hispanic, or Asian), marital status (married, divorced/separate/single, or } \\
\text { widowed); living status (alone or not), family history of diabetes (yes/no), menopausal status (premenopausal or postmenopausal; never, past, or current } \\
\text { menopausal hormone use), oral contraceptive use (never, past, or current use (NHS II only)), alcohol drinking (0, 0.1-4.9, 5.0-14.9, 15.0-19.9, 20.0-29.9, } \\
\text { or } \geq 30 \mathrm{~g} / \text { day), and total energy intake (fifths); all covariates, except ethnicity and family history of diabetes, were time varying. } \\
\text { ^Results were pooled by using random effects model. } \\
\text { tTests for heterogeneity between studies were quantified using Cochrane Q statistic and I }{ }^{2} \text { statistic. } \\
\text { fUnhealthy lifestyles include current smoking, physical activity levels }<30 \mathrm{~min} / \text { day at moderate to vigorous intensity, diet in bottom three fifths of Alternate } \\
\text { Healthy Eating Index score, and body mass index } \geq 25 \text {. }\end{array}$} \\
\hline
\end{tabular}


with age in our previous studies. ${ }^{25} 34$ Another potential explanation for larger effect sizes in the NHS II could be that nearly all women in the NHS, on average at age 55 years, had quit rotating night shift work at study baseline, whereas more than $21 \%$ of women in the NHS II were still in rotating night shift work schedules. Results from previous studies suggested that the deleterious health consequences of shift work decreased with increasing time from when shift work ceased..$^{35} 36$

We observed a higher body mass index and higher smoking rates among women with rotating night shift work, in line with previous epidemiologic studies, including our studies in the NHS and NHSII cohorts. ${ }^{81416}$ However, in this study, we found no appreciable differences in quality of diet and amount of physical activity across categories of duration of rotating night shift work. The evidence for the effect of shift work on physical activity presents a mixed picture. Previous studies have reported negative, positive, or no effects on levels of physical activity in shift workers compared with day workers. ${ }^{141837}$ With respect to quality of diet, a few studies observed alterations in eating habit and specific nutrient intake among shift workers relative to day workers. ${ }^{17-19}$ However, owing to limitations in study design and variability in defining shift work and assessing dietary intake, results are difficult to compare between studies. In our previous study in the NHS II, history of night shift work was not associated with quality of diet, ${ }^{14}$ but no study has investigated the overall diet quality across different shift work categories. Consistent with our previous study and another study among US black women, ${ }^{83}$ adjustment for lifestyle factors did not attenuate the association between rotating night shift work and type 2 diabetes.

Previous evidence on the health effect of lifestyle factors in conjunction with shift work is sparse. In this study, we found that the highest risk for type 2 diabetes was among women with at least 10 years of rotating night shift work duration and at least three unhealthy lifestyle factors compared with women who had no history of rotating night shift work and one or fewer unhealthy lifestyle factors. Unhealthy lifestyle factors were consistently associated with higher risk of type 2 diabetes across each stratum of rotating night shift work duration, emphasizing the importance of a healthy lifestyle in preventing type 2 diabetes. This study is limited to shift work in US female nurses, and the specific shift work pattern examined is rotating night shift work. Shift work patterns can differ between professions and countries and are differentially associated with risk of type 2 diabetes. ${ }^{38} 39$ The generalizability of our findings to other populations with other shift work patterns may be limited, and further studies are warranted to replicate our findings in other populations with rotating night shift work and other shift work patterns.

Potential mechanisms

Rotating night shift work and related unhealthy lifestyles are likely to share several possible mechanisms involved in increasing the risk of type 2 diabetes. For example, rotating night shift work alters sleep and circadian rhythms that play important roles in daily normal metabolic function, by regulating patterns of energy expenditure and hormones involved in energy metabolism such as leptin, ghrelin, thyrotropin, insulin, and melatonin. ${ }^{40-42}$ Disruption of sleep and circadian rhythms could contribute to insulin resistance, impaired glucose regulation, and development of type 2 diabetes. ${ }^{43-46}$ Accumulating evidence also suggests that sleep and circadian rhythms are influenced by lifestyle behaviors such as smoking, ${ }^{47-49}$ diet, ${ }^{50}{ }^{51}$ and physical activity. ${ }^{52} 53$ Recent studies have found that circadian related genetic variants could modify the effect of dietary carbohydrate intake on cardiometabolic traits and the association of sleep duration with body mass index and macronutrient intake. ${ }^{54} 55$ Additionally, given the increasing evidence underlying the role of gut microbiota and microbial metabolites in the development of type 2 diabetes, ${ }^{56-60}$ changes in gut microbiota have been proposed as a potential pathway linking shift work and metabolic diseases because sleep loss and circadian misalignment could disrupt the intestinal microbiota. ${ }^{61-63}$ Furthermore, lifestyle behaviors, especially diet and physical activity, could affect gut microbial diversity and metabolites, ${ }^{64-67}$ which may modify the association between rotating night shift work and risk of type 2 diabetes. Further studies are needed to explore the pathways underlying the interaction between rotating night shift work and lifestyle factors on risk of type 2 diabetes.

\section{Strengths and limitations of study}

The major strengths of our study include the prospective design, large sample size, repeated measures of lifestyle and body weight, and long term follow-up. To our knowledge, this is the first study to investigate how rotating night shift work and modifiable lifestyle factors are jointly related to risk of type 2 diabetes. The study also has certain limitations. Our estimates of joint effect and additive interactions were assessed on a relative scale, derived from the widely used Cox proportional hazards model. The test and measures of interactions depended neither on follow-up time nor on the covariates, but the Cox model relied on the proportional hazards assumption and could be biased, especially if confounding was present. We carefully controlled for confounding, using time varying covariates. In addition, the presence of a statistical additive interaction does not necessarily imply the presence of a causal interaction. The participants were all female nurses and mostly white, which limits the generalizability of our findings to other populations, particularly men and other racial or ethnic groups. However, the relative homogeneity of this study population in educational attainment and socioeconomic status enhances the internal validity of our findings. Because information on rotating night shift work and lifestyles was self reported, potential exists for exposure misclassification. However, all 
our questionnaires were validated, and the high educational status of the study participants ensures that we are obtaining quality data. Furthermore, if measurement error does exist, the prospective design of our study indicates that such bias would likely be random with respect to outcome status, resulting in attenuation of the effect estimates and thus underestimation of the true associations. Additionally, the assessments of rotating night shift work lacked information about actual night working time and work stress, both of which have been shown to be independently associated with risk of type 2 diabetes. ${ }^{68}$

${ }^{69}$ We also did not have data on permanent night shift work over time and could have included participants with such schedules in the reference group. Although the lifestyle factors were differentially associated with risk of type 2 diabetes, we assigned equal weight to each lifestyle factor in our unhealthy lifestyle score. However, our analysis based on a weighted score yielded similar results. Finally, in the NHS, we had only baseline assessment of rotating night shift work.

\section{Conclusion and public health implications}

In two large US cohorts of female nurses, both rotating night shift work and unhealthy lifestyle were associated with a higher risk of type 2 diabetes, and the joint effect was higher than the addition of the risks associated with each individual factor. Our findings suggest that most cases of type 2 diabetes could be prevented by adherence to a healthy lifestyle, and the benefits would be larger in rotating night shift workers. Further studies are warranted to confirm our findings and clarify the underlying mechanisms.

Contributors: ZS, FBH, and SNB were involved in the study conception and design. ZS analyzed and interpreted the data. $Y L, G Z$, and YG provided statistical expertise. ZS drafted the manuscript. All the authors participated in the interpretation of the results and critical revision of the manuscript. The corresponding author attests that all listed authors meet authorship criteria and that no others meeting the criteria have been omitted. ZS and SNB have full access to all of the data in the study and take responsibility for the integrity of the data and the accuracy of the data analysis; they are the guarantors.

Funding: This study was supported by research grants UM1 CA186107, R01 HL034594, R01 HL088521, UM1 CA176726, UM1 CA167552, R01 OH009893, R01 HL35464, P30 DK46200, and R01 DK112940 from the National Institutes of Health (NIH). ZS is supported by the Young Scientists Fund of the National Natural Science Foundation of China (81703214) and the China Postdoctoral Science Foundation (2016M602314). SNB is supported by a career development grant from the NIH (K01 DK107804). The funders had no role in the design and conduct of the study; collection, management, analysis, and interpretation of the data; preparation, review, or approval of the manuscript; or the decision to submit the manuscript for publication.

Competing interests: All authors have completed the ICMJE uniform disclosure form at www.icmje.org/coi_disclosure.pdf (available on request from the corresponding author) and declare: no support from any organization for the submitted work other than those detailed above; no financial relationships with any organizations that might have an interest in the submitted work in the previous three years; no other relationships or activities that could appear to have influenced the submitted work.

Ethical approval: The Institutional Review Boards of Brigham and Women's Hospital and the Harvard T. H. Chan School of Public Health approved this study. Return of the mailed questionnaire was considered to imply informed consent. Protocol number: 2009-P-002375.

Transparency declaration: The lead authors (the manuscript's guarantors) affirm that the manuscript is an honest, accurate, and transparent account of the study being reported; that no important aspects of the study have been omitted; and that any discrepancies from the study as planned (and, if relevant, registered) have been explained.

Data sharing: The statistical code and technical processes are available from the corresponding author at zshan@hsph.harvard.edu.

This is an Open Access article distributed in accordance with the Creative Commons Attribution Non Commercial (CC BY-NC 4.0) license, which permits others to distribute, remix, adapt, build upon this work non-commercially, and license their derivative works on different terms, provided the original work is properly cited and the use is noncommercial. See: http://creativecommons.org/licenses/by-nc/4.0/.

1 Alterman T, Luckhaupt SE, Dahlhamer JM, Ward BW, Calvert GM. Prevalence rates of work organization characteristics among workers in the U.S.: data from the 2010 National Health Interview Survey. Am I Ind Med 2013:56:647-59. doi:10.1002/ajim.22108

2 Wright KPJr, Bogan RK, Wyatt JK. Shift work and the assessment and management of shift work disorder (SWD). Sleep Med Rev 2013;17:41-54. doi:10.1016/j.smrv.2012.02.002

3 Kivimäki M, Batty GD, Hublin C. Shift work as a risk factor for future type 2 diabetes: evidence, mechanisms, implications, and future research directions. PLoS Med 2011;8:e1001138. doi:10.1371/journal.pmed.1001138

4 Labor Force Statistics from the Current Population Survey. Work schedules (flexible and shift schedules). 2018. https://www.bls.gov/ $\mathrm{cps} /$ Ifcharacteristics.htm\#schedules.

5 National Institute for Occupational Safety and Health (NIOSH). NIOSH training for nurses on shift work and long work hours. 2015. https://www.cdc.gov/niosh/docs/2015-115/default.html.

6 Wang F, Zhang L, Zhang Y, et al. Meta-analysis on night shift work and risk of metabolic syndrome. Obes Rev 2014;15:709-20. doi:10.1111/obr.12194

7 Kecklund G, Axelsson J. Health consequences of shift work and insufficient sleep. BMJ 2016;355:i5210. doi:10.1136/bmj.i5210

8 Pan A, Schernhammer ES, Sun Q, Hu FB. Rotating night shift work and risk of type 2 diabetes: two prospective cohort studies in women. PLoS Med 2011;8:e1001141. doi:10.1371/journal. pmed.1001141

9 Hu FB, Manson JE, Stampfer MJ, et al. Diet, lifestyle, and the risk of type 2 diabetes mellitus in women. N Engl J Med 2001;345:790-7. doi:10.1056/NEJMoa010492

10 Pan A, Wang Y, Talaei M, Hu FB, Wu T. Relation of active, passive, and quitting smoking with incident type 2 diabetes: a systematic review and meta-analysis. Lancet Diabetes Endocrinol 2015;3:958-67. doi:10.1016/S2213-8587(15)00316-2

11 Ding D, Lawson KD, Kolbe-Alexander TL, et al, Lancet Physical Activity Series 2 Executive Committee. The economic burden of physical inactivity: a global analysis of major non-communicable diseases. Lancet 2016;388:1311-24. doi:10.1016/S01406736(16)30383-X

12 Li G, Zhang P, Wang J, et al. The long-term effect of lifestyle interventions to prevent diabetes in the China Da Qing Diabetes Prevention Study: a 20-year follow-up study. Lancet 2008;371:1783-9. doi:10.1016/S0140-6736(08)60766-7

13 Liu Q, Shi J, Duan P, et al. Is shift work associated with a higher risk of overweight or obesity? A systematic review of observational studies with meta-analysis. Int J Epidemiol 2018. doi:10.1093/ije/dyy079

14 Ramin C, Devore EE, Wang W, Pierre-Paul J, Wegrzyn LR, Schernhammer ES. Night shift work at specific age ranges and chronic disease risk factors. Occup Environ Med 2015;72:100-7. doi:10.1136/oemed-2014-102292

15 Buchvold HV, Pallesen S, Øyane NM, Bjorvatn B. Associations between night work and BMI, alcohol, smoking, caffeine and exercise--a cross-sectional study. BMC Public Health 2015;15:1112. doi:10.1186/s12889-015-2470-2

16 Nea FM, Kearney J, Livingstone MB, Pourshahidi LK, Corish CA Dietary and lifestyle habits and the associated health risks in shift workers. Nutr Res Rev 2015;28:143-66. doi:10.1017/ S095442241500013X

17 Morikawa Y, Miura K, Sasaki S, et al. Evaluation of the effects of shift work on nutrient intake: a cross-sectional study. J Occup Health 2008;50:270-8. doi:10.1539/joh.L7116

18 Esquirol Y, Bongard V, Mabile L, Jonnier B, Soulat IM, Perret B. Shift work and metabolic syndrome: respective impacts of job strain, physical activity, and dietary rhythms. Chronobiol Int 2009;26:54459. doi:10.1080/07420520902821176

19 Ferri GM, Cavone D, Intranuovo G, Macinagrossa L. Healthy Diet And Reduction Of Chronic Disease Risks Of Night Shift Workers. Curr Med Chem 2017. doi:10.2174/0929867324666170720160632

20 Willett WC, Sampson L, Stampfer MJ, et al. Reproducibility and validity of a semiquantitative food frequency questionnaire. Am J Epidemiol 1985;122:51-65. doi:10.1093/oxfordjournals.aje. a114086 
21 Chiuve SE, Fung TT, Rimm EB, et al. Alternative dietary indices both strongly predict risk of chronic disease. J Nutr 2012;142:1009-18. doi:10.3945/jn.111.157222

$22 \mathrm{Hu}$ FB, Sigal RJ, Rich-Edwards JW, et al. Walking compared with vigorous physical activity and risk of type 2 diabetes in women: a prospective study. JAMA 1999;282:1433-9. doi:10.1001/ jama.282.15.1433

23 Wolf AM, Hunter DJ, Colditz GA, et al. Reproducibility and validity of a self-administered physical activity questionnaire. Int J Epidemiol 1994;23:991-9. doi:10.1093/ije/23.5.991

24 Rimm EB, Stampfer MJ, Colditz GA, Chute CG, Litin LB, Willett WC. Validity of self-reported waist and hip circumferences in men and women. Epidemiology 1990;1:466-73. doi:10.1097/00001648-199011000-00009

25 LiY, Ley SH, Tobias DK, et al. Birth weight and later life adherence to unhealthy lifestyles in predicting type 2 diabetes: prospective cohort study. BMJ 2015;351:h3672. doi:10.1136/bmj.h3672

26 National Diabetes Data Group. Classification and diagnosis of diabetes mellitus and other categories of glucose intolerance. Diabetes 1979;28:1039-57. doi:10.2337/diab.28.12.1039

27 Report of the Expert Committee on the Diagnosis and Classification of Diabetes Mellitus. Diabetes Care 1997;20:1183-97. doi:10.2337/diacare.20.7.1183

28 Manson JE, Rimm EB, Stampfer MJ, et al. Physical activity and incidence of non-insulin-dependent diabetes mellitus in women. Lancet 1991;338:774-8. doi:10.1016/0140-6736(91)90664-B

29 VanderWeele TJ, Asomaning K, Tchetgen Tchetgen E), et al. Genetic variants on 15q25.1, smoking, and lung cancer: an assessment of mediation and interaction. Am I Epidemiol 2012:175:1013-20. doi:10.1093/aje/kwr467

30 Li R, Chambless L. Test for additive interaction in proportional hazards models. Ann Epidemiol 2007:17:227-36. doi:10.1016/j.annepidem.2006.10.009

31 VanderWeele TJ, Tchetgen Tchetgen EJ. Attributing effects to interactions. Epidemiology 2014;25:711-22. doi:10.1097/EDE.0000000000000096

32 Klein S, Sheard NF, Pi-Sunyer X, et al, American Diabetes Association, North American Association for the Study of Obesity, American Society for Clinical Nutrition. Weight management through lifestyle modification for the prevention and management of type 2 diabetes: rationale and strategies: a statement of the American Diabetes Association, the North American Association for the Study of Obesity, and the American Society for Clinical Nutrition. Diabetes Care 2004;27:2067-73. doi:10.2337/diacare.27.8.2067

33 Vimalananda VG, Palmer JR, Gerlovin H, et al. Night-shift work and incident diabetes among African-American women. Diabetologia 2015;58:699-706. doi:10.1007/s00125-014-3480-9

34 Cohen L, Curhan GC, Forman JP. Influence of age on the association between lifestyle factors and risk of hypertension. J Am Soc Hypertens 2012;6:284-90. doi:10.1016/j.jash.2012.06.002

35 Vetter C, Devore EE, Wegrzyn LR, et al. Association Between Rotating Night Shift Work and Risk of Coronary Heart Disease Among Women. JAMA 2016;315:1726-34. doi:10.1001/jama.2016.4454

36 Guo Y, Liu Y, Huang X, et al. The effects of shift work on sleeping quality, hypertension and diabetes in retired workers. PLOS One 2013;8:e71107. doi:10.1371/journal.pone.0071107

37 Bushnell PT, Colombi A, Caruso CC, Tak S. Work schedules and health behavior outcomes at a large manufacturer. Ind Health 2010;48:395-405. doi:10.2486/indhealth.MSSW-03

38 Vetter C, Dashti HS, Lane JM, et al. Night Shift Work, Genetic Risk, and Type 2 Diabetes in the UK Biobank. Diabetes Care 2018;41:762-9. doi:10.2337/dc17-1933

39 Hansen AB, Stayner L, Hansen J, Andersen ZJ. Night shift work and incidence of diabetes in the Danish Nurse Cohort. Occup Environ Med 2016;73:262-8. doi:10.1136/ oemed-2015-103342

40 Markwald RR, Melanson EL, Smith MR, et al. Impact of insufficient sleep on total daily energy expenditure, food intake, and weight gain. Proc Natl Acad Sci U S A 2013;110:5695-700. doi:10.1073/pnas.1216951110

41 McHill AW, Melanson EL, Higgins J, et al. Impact of circadian misalignment on energy metabolism during simulated nightshift work. Proc Natl Acad Sci U S A 2014;111:17302-7. doi:10.1073/pnas.1412021111

42 Depner CM, Stothard ER, Wright KPJr. Metabolic consequences of sleep and circadian disorders. Curr Diab Rep 2014;14:507. doi:10.1007/s11892-014-0507-z

43 Shan Z, Ma H, Xie M, et al. Sleep duration and risk of type 2 diabetes: a meta-analysis of prospective studies. Diabetes Care 2015;38:529-37. doi:10.2337/dc14-2073

44 Leproult R, Holmbäck U, Van Cauter E. Circadian misalignment augments markers of insulin resistance and inflammation, independently of sleep loss. Diabetes 2014;63:1860-9. doi:10.2337/db13-1546
45 Li Y, Gao X, Winkelman JW, et al. Association between sleeping difficulty and type 2 diabetes in women. Diabetologia 2016;59:719-27 doi:10.1007/s00125-015-3860-9

46 McHill AW, Wright KPJr. Role of sleep and circadian disruption on energy expenditure and in metabolic predisposition to human obesity and metabolic disease. Obes Rev 2017;18(Suppl 1):15-24. doi:10.1111/obr.12503

47 Barateau L, Jaussent I, Lopez R, et al. Smoking Alcohol, Drug Use, Abuse and Dependence in Narcolepsy and Idiopathic Hypersomnia: A Case-Control Study. Sleep 2016;39:573-80. doi:10.5665/ sleep. 5530

48 Jimenez-Ruiz CA, Sculier JP, Lundback B, et al, ERS Tobacco Control Committee. Smoking Control Is a Priority to Promote Heart, Lung, Blood, and Sleep Health. Am J Respir Crit Care Med 2015;191:1471-3. doi:10.1164/rccm.201504-0746LE

49 Bellatorre A, Choi K, Lewin D, Haynie D, Simons-Morton B. Relationships Between Smoking and Sleep Problems in Black and White Adolescents. Sleep 2017;40. doi:10.1093/sleep/zsw031

50 Kentish SJ, Vincent AD, Kennaway DJ, Wittert GA, Page AJ. High-Fat Diet-Induced Obesity Ablates Gastric Vagal Afferent Circadian Rhythms. J Neurosci 2016;36:3199-207. doi:10.1523/JNEUROSCI.2710-15.2016

51 Pivovarova O, Jürchott K, Rudovich N, et al. Changes of Dietary Fat and Carbohydrate Content Alter Central and Peripheral Clock in Humans. J Clin Endocrinol Metab 2015;100:2291-302. doi:10.1210/jc.2014-3868

52 Mustian KM, Sprod LK, Janelsins M, et al. Multicenter, randomized controlled trial of yoga for sleep quality among cancer survivors. J Clin Oncol 2013;31:3233-41. doi:10.1200/JCO.2012.43.7707

53 Chaput JP, Saunders TJ, Carson V. Interactions between sleep, movement and other non-movement behaviours in the pathogenesis of childhood obesity. Obes Rev 2017;18(Suppl 1):7-14. doi:10.1111/obr.12508

54 Dashti HS, Follis JL, Smith CE, et al, CHARGE Nutrition Study Group. Gene-Environment Interactions of Circadian-Related Genes for Cardiometabolic Traits. Diabetes Care 2015;38:1456-66. doi:10.2337/dc14-2709

55 Dashti HS, Follis JL, Smith CE, et al. Habitual sleep duration is associated with $\mathrm{BMI}$ and macronutrient intake and may be modified by CLOCK genetic variants. Am J Clin Nutr 2015;101:135-43. doi:10.3945/ajcn.114.095026

56 Bouter KE, van Raalte DH, Groen AK, Nieuwdorp M. Role of the Gut Microbiome in the Pathogenesis of Obesity and Obesity-Related Metabolic Dysfunction. Gastroenterology 2017;152:1671-8. doi:10.1053/j.gastro.2016.12.048

57 Pedersen HK, Gudmundsdottir V, Nielsen HB, et al, MetaHIT Consortium. Human gut microbes impact host serum metabolome and insulin sensitivity. Nature 2016;535:376-81. doi:10.1038 nature 18646

58 Komaroff AL. The Microbiome and Risk for Obesity and Diabetes. JAMA 2017;317:355-6. doi:10.1001/jama.2016.20099

59 Shan Z, Sun T, Huang H, et al. Association between microbiotadependent metabolite trimethylamine- $\mathrm{N}$-oxide and type 2 diabetes. Am J Clin Nutr 2017; 106:888-94.

60 Lynch SV, Pedersen 0. The Human Intestinal Microbiome in Health and Disease. N Engl J Med 2016;375:2369-79. doi:10.1056/NEJMra1600266

61 Leone V, Gibbons SM, Martinez K, et al. Effects of diurnal variation of gut microbes and high-fat feeding on host circadian clock function and metabolism. Cell Host Microbe 2015;17:681-9. doi:10.1016/j.chom.2015.03.006

62 Benedict C, Vogel H, Jonas W, et al. Gut microbiota and glucometabolic alterations in response to recurrent partial sleep deprivation in normal-weight young individuals. Mol Metab 2016:5:1175-86. doi:10.1016/i.molmet.2016.10.003

63 Reynolds AC, Paterson JL, Ferguson SA, Stanley D, Wright KPJr, Dawson D. The shift work and health research agenda: Considering changes in gut microbiota as a pathway linking shift work, sleep loss and circadian misalignment, and metabolic disease. Sleep Med Rev 2017;34:3-9. doi:10.1016/i.smrv.2016.06.009

64 Shoaie S, Ghaffari P, Kovatcheva-Datchary P, et al, MICRO-Obes Consortium. Quantifying Diet-Induced Metabolic Changes of the Human Gut Microbiome. Cell Metab 2015;22:320-31. doi:10.1016/i.cmet.2015.07.001

65 Clarke SF, Murphy EF, O’Sullivan O, et al. Exercise and associated dietary extremes impact on gut microbial diversity. Gut 2014;63:1913-20. doi:10.1136/gutjnl-2013-306541

66 Sonnenburg JL, Bäckhed F. Diet-microbiota interactions as moderators of human metabolism. Nature 2016;535:56-64. doi:10.1038/nature18846 
67 Aron-Wisnewsky J, Clément K. The gut microbiome, diet, and links to cardiometabolic and chronic disorders. Nat Rev Nephrol 2016;12:169-81. doi:10.1038/nrneph.2015.191

68 Kivimaki M, Virtanen M, Kawachi I, et al. Long working hours, socioeconomic status, and the risk of incident type 2 diabetes: a meta-analysis of published and unpublished data from 222120 individuals. Lancet Diabetes Endocrinol 2015;3:27-34
69 Brunner EJ, Kivimäki M. Epidemiology: work-related stress and the risk of type 2 diabetes mellitus. Nat Rev Endocrinol 2013;9:449-50. doi:10.1038/nrendo.2013.124

Supplementary tables and figures 